\title{
THE LATTICE OF TOPOLOGIES: STRUCTURE AND COMPLEMENTATION
}

\author{
A. K. STEINER(1)
}

The family of all topologies on a set is a complete, bounded latlice. The purpose of this paper is to study the structure of the lattice of topologies, employing the notion of ultraspace introduced by Fröhlich [7] and to show that this lattice is complemented.

The set of ultraspaces may naturally be divided into two classes, each of which generates a sublattice. One of these sublattices is the lattice of $T_{1}$-topologies. The other, which is studied in $\$ 2$, is the lattice of principal topologies. Principal topologies are defined in terms of ultraspaces and are then characterized by properties of open sets.

Some topological properties of ultraspaces are investigated in $\$ 4$ and maximal regular (maximal $T_{1}$, maximal normal, etc.) topologies are characterized in terms of ultraspaces.

The problem of complementation in the lattice of topologies has been outstanding for some time. However, several partial solutions have been provided. Hartmanis [11] first showed the lattice was complemented if the ground set was finite and asked whether this was true in the infinite case. Gaifman [8] gave a positive answer for denumerable sets and Berri [3], using this fact, was able to provide complements for certain special topologies such as a topological group with a dense, nonopen countable subgroup.

It is shown in $\$ 5$ that the lattice of principal topologies is complemented. Gaifman [9] established that the complementation problem can be reduced to verifying that each $T_{1}$-topology has a complement. In $\S 6$, it is proved that if every $T_{1}$-topology has a lattice complement which is a principal topology, then every topology does. In $\$ 7$ it is shown that the lattice of topologies on an arbitrary set is complemented by proving that every topology in the sublattice of $T_{1}$-topologies has a lattice complement which lies in the sublattice of principal topologies.

Preliminary definitions and remarks. Throughout this paper, $E$ will denote an arbitrary set, and $\tau$, with or without subscripts, will denote a topology on $E$,

Received by the editors September 27, 1965.

(1) The results in this paper are contained in the author's doctoral dissertation presented to the Department of Mathematics, University of New Mexico in 1965. This research was partially supported by the National Science Foundation, Grant GP-2214. 
whose members are open sets. The symbol $|E|$ will denote the cardinality of the set $E$.

The definitions of $T_{0}, T_{1}, T_{2}$, regular, completely regular and normal topologies are those found in Kelley [12]. The definitions of partially ordered set, lattice, sublattice, infimum and supremum are those found in Szász [16] and Birkhoff [5]. The definitions and properties of filters and ultrafilters are those of Cartan [6], Samuel [14] and Schmidt [15].

The family $\Sigma$ of all topologies definable on a set $E$, partially ordered by set inclusion, is a complete lattice (Vaidyanathaswamy [17]). The greatest element of $\Sigma$, the discrete topology, will be denoted by 1 and the least element, the trivial topology, by 0 .

A topology $\tau$ on $E$ is an ultraspace if the only topology on $E$ strictly finer than $\tau$ is the discrete topology.

For a filter $\mathscr{F}$ on $E$, Fröhlich [7] defined $S(p, \mathscr{F})$ to be the family of sets $\mathfrak{P}(E-\{p\}) \cup \mathscr{F}$, where $\mathfrak{P}(E-\{p\})$ is the collection of all subsets of $E$ which do not contain $p$. Hence, $\mathfrak{S}(p, \mathscr{F})$ is a topology on $E$ such that for every $x \in E$, $x \neq p$, the set $\{x\}$ is open and the open sets containing $p$ are just the sets in $\mathscr{F}$ which contain $p$.

Fröhlich proved that the ultraspaces on $E$ are exactly the topologies of the form $\subseteq(x, \mathscr{U})$ where $x \in E$ and $\mathscr{U}$ is an ultrafilter on $E, \mathscr{U} \neq \mathscr{U}(x)$; that the correspondence between ultraspaces and ordered pairs $(x, \mathscr{U})$ is one-to-one; and that every topology $\tau$ on $E$ is the infimum of the ultraspaces on $E$ which are finer than $\tau$.

Since an ultrafilter is either principal or nonprincipal (i.e. it either contains a finite set or it does not), the class of ultraspaces on $E$ is composed of the class of nonprincipal ultraspaces (those in which the ultrafilter is nonprincipal) and the class of principal ultraspaces (those in which the ultrafilter is principal).

A point $x \in E$ is called an isolated point of $\tau$ if $\{x\} \in \tau$. If $x_{0}$ is not an isolated point, then $\tau \leqq \subseteq\left(x_{0}, \mathscr{U}\right)$ for some ultrafilter $\mathscr{U}$.

An infraspace is a topology such that the only topology strictly coarser than it is the trivial topology. Every infraspace has the form $\{E, A, \varnothing\}$ where $A \subset E$, $A \neq \varnothing, A \neq E$. Clearly every topology $\tau$ is the supremum of infraspaces coarser than $\tau$.

1. $T_{1}$-topologies. A topology on $E$ is a $T_{1}$-topology if for every $x \in E$, the set $\{x\}$ is closed. For every $x \in E$ and for every nonprincipal ultrafilter $\mathscr{U}$ on $E$, the set $E-\{x\} \in \mathscr{U}$. Hence $\{x\}$ is closed in every nonprincipal ultraspace. In a principal ultraspace $\mathfrak{S}(x, \mathscr{U}(y))$, the set $\{y\}$ is not closed. Therefore, an ultraspace is a $T_{1}$-topology if and only if it is a nonprincipal ultraspace.

THEOREM 1.1. A topology $\tau$ on $E$ is a $T_{1}$-topology if and only if it is the infimum of nonprincipal ultraspaces. 
Proof. Any topology finer than a $T_{1}$-topology must also be a $T_{1}$-topology; so a $T_{1}$-topology can be the infimum of only nonprincipal ultraspaces.

For each $x \in E$, the set $E-\{x\}$ is open in each nonprincipal ultraspace, and thus in the infimum of any family of nonprincipal ultraspaces, so $\{x\}$ is closed in this infimum.

The $T_{1}$-topologies form a lattice $\Lambda$, which is a complete sublattice of $\Sigma$, and which has been studied previously by Bagley [1], and Hartmanis [11]. The finest $T_{1}$-topology is the discrete topology and the coarsest is the cofinite topology $\mathscr{C}$, in which a nonempty set is open if and only if its complement is a finite set. On a finite set the only $T_{1}$-topology is the discrete topology.

2. Principal topologies. Every topology $\tau$ on $E$ is the infimum of all ultraspaces on $E$ finer than $\tau$. If also $\tau=\inf \{\mathfrak{S}(x, \mathscr{U}(y)) \mid \tau \leqq \mathfrak{S}(x, \mathscr{U}(y))\}$, then $\tau$ is said to be a principal topology. If $x=y$, then $\mathfrak{S}(x, \mathscr{U}(y))$ is the discrete topo$\log y$ and if $x \neq y$, then $\mathfrak{S}(x, \mathscr{U}(y))$ is a principal ultraspace.

A principal ultraspace $\mathfrak{S}(p, \mathscr{U}(q))$ on $E$ is a principal topology in which every open set containing $p$ must also contain $q$.

The topology $\tau=\mathfrak{S}(p, \mathscr{U}(q)) \wedge \mathfrak{S}(q, \mathscr{U}(r))$ is a principal topology in which for each $x \in E, x \neq p, x \neq q$, the set $\{x\} \in \tau$. Every open set $p$ must contain $q$ and every open set containing $p$ must also contain $r$. Therefore $\tau \leqq \subseteq(p, \mathscr{U}(r))$.

THEOREM 2.1. If $\tau$ is a principal topology on E, the set

$$
B_{x}=\{y \in E \mid \Im(x, \mathscr{U}(y)) \geqq \tau\}
$$

is open for each $x \in E$.

Proof. If $\{x\} \in \tau$ then $B_{x}=\{x\}$, since if there is a $y \in E, y \neq x$, such that $\tau \leqq \Im(x, \mathscr{U}(y))$, then $\{x\} \notin \tau$. Let $\tau \leqq \Im(p, \mathscr{U}(q))$. If $p \notin B_{x}$, then $B_{x}$ is open in $\mathfrak{S}(p, \mathscr{U}(q))$. If $p \in B_{x}$, then $\tau \leqq \Im(x, \mathscr{U}(p)) \wedge \mathfrak{S}(p, \mathscr{U}(q)) \leqq \Im(x, \mathscr{U}(q))$ (Fröhlich [7]). Hence $q \in B_{x}$ and $B_{x}$ is open in $\Im(p, \mathscr{U}(q))$. Since $B_{x}$ is open in each $\mathfrak{S}(p, \mathscr{U}(q))$ finer than $\tau, B_{x} \in \tau$.

THEOREM 2.2. If $\tau$ is a principal topology on $E$, then for each $x \in E$, every set in $\tau$ containing $x$ must also contain the set

$$
B_{x}=\{y \in E \mid \mathfrak{S}(x, \mathscr{U}(y)) \geqq \tau\} .
$$

Proof. If $S$ is a set containing $x$ and $y \notin S$ for some $y \in B_{x}$, then $S$ is not in $\Im(x, \mathscr{U}(y))$. Since $\tau \leqq \Im(x, \mathscr{U}(y)), S$ is not in $\tau$.

A subcollection $\mathscr{B}$ of $\tau$ is a base of open sets minimal at each point if for every $x \in E$ there is a $V \in \mathscr{B}$ such that $x \in V$ and every set in $\tau$ containing $x$ must contain $V$.

THEOREM 2.3. A topology $\tau$ is a principal topology if and only if it has a base of open sets minimal at each point. 
Proof. Let $\tau$ be a principal topology. For each $x \in E$, the set

$$
B_{x}=\{y \in E \mid \Im(x, \mathscr{U}(y)) \geqq \tau\}
$$

is open (Theorem 2.1) and every set in $\tau$ containing $x$ must contain $B_{x}$ (Theorem 2.2).

Suppose $\tau$ is not a principal topology. Then there is a nonprincipal ultraspace $\mathfrak{S}(x, \mathscr{U})$ such that $\tau \leqq \mathfrak{S}(x, \mathscr{U})$. If there is a minimal open set $V$ containing $x$, then $V \in \mathscr{U}$ and every open set containing $x$ must contain $V$. Hence $\tau \leqq \Im(x, \mathscr{U}(y))$ for all $y \in V$ and $\tau \leqq \bigwedge\{\Im(x, \mathscr{U}(y)) \mid y \in V\} \leqq \Im(x, \mathscr{U})$. Thus, if $\tau$ has a base of open sets minimal at each point, then for each nonprincipal ultraspace $\mathfrak{S}(x, \%)$ finer than $\tau$ there is a principal topology finer than $\tau$ and coarser than $\mathfrak{S}(x, \mathcal{M})$ which implies $\tau$ is a principal topology. Therefore, a nonprincipal topology can not have a base of open sets minimal at each point.

THEOREM 2.4. A topology $\tau$ is a principal topology if and only if arbitrary intersections of open sets are open.

Proof. If arbitrary intersections of open sets are open, for each $x \in E$ the intersection of all open sets containing $x$ is a minimal open set at $x$ and the family $\mathscr{B}$ of open sets minimal at $x$ for all $x \in E$, is a base for $\tau$. Hence $\tau$ is a principal topology.

If $\tau$ is a principal topology and $\mathscr{N}$ is a subcollection of $\tau$, then $\bigcap \mathscr{N}$ is open since for each $x \in \bigcap \mathscr{N}$, the minimal open set containing $x$ is also in $\bigcap \mathscr{N}$.

Corollary. An infraspace is a principal topology.

THEOREM 2.5. The principal topologies form a sublattice of the lattice $\mathfrak{Z}$.

Proof. If $\tau_{1}$ and $\tau_{2}$ are principal topologies, then certainly $\tau_{1} \wedge \tau_{2}$ is a principal topology. For each $x$, the set $U_{x}=V_{x}^{1} \cap V_{x}^{2}$, where $V_{x}^{i}$ is a minimal open set in $\tau_{i}$ containing $x$ for $i=1,2$, is a minimal open set in $\tau_{1} \vee \tau_{2}$ containing $x$ and the family $\mathscr{B}=\left\{U_{x} \mid U_{x} \in \tau_{1} \vee \tau_{2}\right.$ is minimal at $x$, for all $\left.x \in E\right\}$ is a base for $\tau_{1} \vee \tau_{2}$, minimal at each point. Hence $\tau_{1} \vee \tau_{2}$ is a principal topology.

The lattice of principal topologies on $E$ will be denoted by $\Pi$. "

The lattice $\Pi$ is a complete lattice since $\Pi$ is meetl complete and is bounded above by 1 . For any subset $\mathscr{H} \subset \Pi, \inf _{\Sigma} \mathscr{H}=\inf _{\Pi} \mathscr{H}$. However, $\Pi$ is not a complete sublattice of $\Sigma$. Let $\tau$ be a $T_{1}$-ultraspace, $\tau \neq 1$. Then $\tau=\sup \left\{\gamma_{i} \mid i \in \Gamma\right\}$, where each $\gamma_{i}, i \in \Gamma$, is an infraspace coarser than $\tau$. Each $\gamma_{i}$ is a principal topology and $\tau=\sup _{\Sigma}\left\{\gamma_{i} \mid i \in \Gamma\right\} \leqq \sup _{\Pi}\left\{\gamma_{i} \mid i \in \Gamma\right\} . \operatorname{But}_{\sup _{\Pi}}\left\{\gamma_{i} \mid i \in \Gamma\right\}$, being finer than a $T_{1}$-topology, must also be a $T_{1}$-topology, so $\sup _{n}\left\{\gamma_{2} \mid i \in \Gamma\right\}=1 \neq \tau$.

A subset $G$ of $E \times E$ is a preorder relation if and only if

(1) $(x, x) \in G$ for all $x \in E$; and

(2) $(x, y) \in G$ and $(y, z) \in G$ imply $(x, z) \in G$ for all $x, y, z \in E$.

Subsequently, the notation $x G y$ will be used to denote that $(x, y) \in G$. 
There is a chain of length $n$ from $x$ to $y$ in $G$ if and only if there are elements $x=x_{0}, x_{1}, \cdots, x_{n-1}, x_{n}=y, n \geqq 0$, such that $x_{0} G x_{1}, x_{1} G x_{2}, \cdots, x_{n-1} G x_{n}$.

Operations $\wedge$ and $\vee$ are defined on preorder relations by

(1) $G_{1} \wedge G_{2}=G_{1} \cap G_{2}$,

(2) $G_{1} \vee G_{2}=\left(G_{1} \cup G_{2}\right)$, where $\hat{G}=\{(x, y) \mid$ there is a chain of length $n$ from $x$ to $y$ in $G, n \geqq 0\}$.

Under these operations, the family $\mathscr{G}$ of preorder relations, partially ordered by set inclusion, is a lattice.

Each preorder relation $G$ defines a topology $\tau_{G}$ : a set $S \subseteq E$ is open if and only if for each $x \in S$, if $x G y$ then $y \in S$. Moreover, the topology $\tau_{G}$ determined by the preorder relation $G$ is a principal topology and $\tau_{G}=\inf \{\circlearrowleft(x, \mathscr{U}(y)) \mid x G y\}$. Each principal topology $\tau$ determines a preorder relation $G_{\tau}$ by $G_{\tau}=\{(x, y) \mid \tau \leqq \mathfrak{S}(x, \mathscr{U}(y))\}$.

THEOREM 2.6. The lattice $\Pi$ of principal topologies is anti-isomorphic to the lattice $\mathscr{G}$ of preorder relations.

Proof. There is a one-to-one correspondence between principal topologies in $\Pi$ and preorder relations in $\mathscr{G}$ since the mappings $\eta: \Pi \rightarrow \mathscr{G}$ and $\phi: \mathscr{G} \rightarrow \Pi$ defined by

$$
\eta(\tau)=G_{\tau}=\{(x, y) \mid \Im(x, \mathscr{U}(y)) \geqq \tau\}
$$

and

$$
\phi(G)=\tau_{G}=\inf \{\Im(x, \mathscr{U}(y)) \mid x G y\}
$$

are inverses. That is, $\eta(\phi(G))=G$ and $\phi(\eta(\tau))=\tau$.

Since $x G_{\tau} y$ holds if and only if $\tau \leqq \Im(x, \mathscr{U}(y))$, if $\tau_{1} \leqq \tau_{2}$ then $G_{\tau_{1}} \geqq G_{\tau_{2}}$. And since $\tau_{G}=\bigwedge\{\Im(x, \mathscr{U}(y)) \mid x G y\}$, then $G_{1} \leqq G_{2}$ implies $\tau_{G_{1}} \geqq \tau_{G_{2}}$. Hence $\eta$ and $\eta^{-1}=\phi$ are antitone.

Thus, the lattice of preorder relations is a complete lattice (since $\Pi$ is) with a least element, namely the relation $\Delta=\{(x, x) \mid x \in E\}$ corresponding to the discrete topology and a greatest element $E \times E$ corresponding to the trivial topology.

Let $G$ be a relation on $E$. There is a path from $x_{0}$ to $x_{n}$ in $G$ if there is a finite collection of elements $x_{1}, x_{2}, \cdots, x_{n-1}$ of $E$ such that $x_{i} G x_{i-1}$ or $x_{i-1} G x_{i}$, $i=1,2, \cdots, n$. If there is a path from $x_{0}$ to $x_{n}$, then there is a path from $x_{n}$ to $x_{0}$.

A subset $F \subseteq E$ is a component of $E$ relative to a relation $G$ if for every pair $x, y \in F$ there is a path from $x$ to $y$ in $G$ and for $w \notin F$ there is no path from $x$ to $w$ for any $x \in F$.

Two distinct components of $E$ relative to $G$ are disjoint and every point of $E$ is in some component.

A relation $\mathrm{Y} G$ on $E$ is connected if $E$ is a component relative to $G$.

A topology $\tau$ on $E$ is connected if $E$ is not the union of two nonempty, disjoint open isets. 
THEOREM 2.7. A principal topology $\tau_{G}$ is connected if and only if $G$ is a connected relation.

Proof. Suppose $\tau_{G}$ is not connected. Then $E=U \cup V$ where $U, V \in \tau_{G}$, $U \neq \varnothing, V \neq \varnothing$ and $U \cap V=\varnothing$. Suppose $G$ is a connected relation. Let $x \in U$ and $y \in V$. Then there is a finite subset $\left\{x=x_{1}, x_{2}, \cdots, x_{n}=y\right\} \subset E$ such that $x_{i} G x_{i+1}$ or $x_{i+1} G x_{i}$ for $i=1,2, \cdots, n-1$. Denote by $x_{k}$ the first $x_{i}$ such that $x_{i} \in V$. Since $x_{1} \in U, k>1$. Thus $x_{k-1} \in U$ and $x_{k} \in V$. If $x_{k-1} G x_{k}$, then $U$ is not open in $\tau_{G}$ and if $x_{k} G x_{k-1}$, then $V$ is not open in $\tau_{G}$. This is a contradiction so $G$ is not a connected relation.

Suppose $G$ is not a connected relation. For some $x_{0} \in E$ let $U=\{y \in E \mid$ there is a path between $x_{0}$ and $\left.y\right\}$. The set $U$ is open. For each $x \in U$, the set $\{y \in E \mid x G y\} \subset U$ because if there is a path between $x_{0}$ and $y$ and $y G z$ holds, then there is a path between $x_{0}$ and $z$. Since $G$ is not connected there is some $y_{0} \in E-U$ and $E-U$ is also open. Otherwise for some $y \in E-U, y G z$ and $z \in U$, but since there is a path between $x_{0}$ and $z$ and $y G z$, then there is a path between $x_{0}$ and $y$. Thus $E-U$ must be open, and $\tau$ is not connected.

A topology on $E$ which is neither a $T_{1}$-topology nor a principal topology is a mixed topology. A mixed topology can be represented as the infimum of a $T_{1}$-topology and a principal topology, but this representation need not be unique. The supremum of two mixed topologies can be a $T_{1}$-topology or a principal topology. For example, if $\tau=\mathfrak{S}(x, \mathscr{U}) \wedge \mathfrak{S}(p, \mathscr{U}(q)), \tau^{\prime}=\mathfrak{S}(x, \mathscr{U}) \wedge \mathfrak{S}(q, \mathscr{U}(p))$, and $\tau^{\prime \prime}=\mathfrak{S}(y, \mathscr{V}) \wedge \mathfrak{S}(p, \mathscr{U}(q))$ where $x \neq y$ and $\mathscr{U} \neq \mathscr{V}$, then $\tau \vee \tau^{\prime}=\mathfrak{S}(x, \mathscr{U})$ and $\tau \vee \tau^{\prime \prime}=\mathfrak{S}(p, \mathscr{U}(q))$. The infimum of two mixed topologies cannot be a $T_{1}$-topology, but it can be a principal topology.

Let $\mathscr{U}$ and $\mathscr{V}$ be distinct nonprincipal ultrafilters. Then there is a set $A$ such that $A \in \mathscr{U}$ and $E-A \in \mathscr{V}$. Let $\tau_{1}=\bigwedge\{\Im(x, \mathscr{U}(q)) \mid q \in A-\{x\}\} \quad$ and $\tau_{2}=\bigwedge\{\Im(y, \mathscr{U}(q)) \mid q \in E-A-\{x\}\}$. Then $\tau_{1} \leqq \Im(x, \mathscr{U})$ and $\tau_{2} \leqq \Im(y, \mathscr{V})$, and $\mathfrak{S}(x, \mathscr{U}) \wedge \tau_{2}$ and $\mathfrak{S}(y, \mathscr{V}) \wedge \tau_{1}$ are mixed topologies. But

$\left[\Im(x, \mathscr{U}) \wedge \tau_{2}\right] \wedge\left[\Im(y, \mathscr{V}) \wedge \tau_{1}\right]=\left[\Im(x, \mathscr{U}) \wedge \tau_{1}\right] \wedge\left[\Im(y, \mathscr{V}) \wedge \tau_{2}\right]=\tau_{1} \wedge \tau_{2}$,

which is a principal topology.

The reader who is interested only in the complementation of $\Sigma$, may skip $\S \S 3,4$ and 5 .

3. Lattice properties of $\Sigma$. Vaidyanathaswamy [17] gave an example to show that the lattice of topologies on a set of cardinality $c$ is not distributive. The following proof uses ultraspaces and is very straightforward.

THEOREM 3.1. The lattice of topologies $\Sigma$ on a set $E$ is distributive if $E$ has fewer than three elements. If $E$ has three or more elements, $\Sigma$ is not even modular. 
Proof. If $E$ has one element or two elements, $\Sigma$ is a distributive lattice. Let $E=\{p, q, r\}$ and let $\mathscr{K}=\Im(p, \mathscr{U}(q)) \wedge \mathfrak{S}(p, \mathscr{U}(r)), \mathscr{L}=\mathfrak{S}(p, \mathscr{U}(r))$, $\mathscr{N}=\mathfrak{S}(r, \mathscr{U}(q))$ be topologies on $E$. Thus $\mathscr{K} \leqq \mathscr{L}$. But $(\mathscr{K} \vee \mathscr{N}) \wedge \mathscr{L}$ $=1 \wedge \mathscr{L}=\mathscr{L}$ and $\mathscr{K} \vee(\mathscr{N} \wedge \mathscr{L}) \leqq \mathfrak{S}(p, \mathscr{U}(q))$ since $\mathscr{K} \leqq \mathfrak{S}(p, \mathscr{U}(q))$ and $\mathscr{N} \wedge \mathscr{L} \leqq \subseteq(p, \mathscr{U}(q))$. Thus $(\mathscr{K} \vee \mathscr{N}) \wedge \mathscr{L} \$ \mathscr{K} \vee(\mathscr{N} \wedge \mathscr{L})$ since $\mathscr{L} \$ \subseteq(p, \mathscr{U}(q))$ and $\Sigma$ is not modular.

A lattice $L$ is self-dual if and only if there is a one-to-one mapping $\phi$ of $L$ onto itself such that $\phi(a \wedge b)=\phi(a) \vee \phi(b)$ and $\phi(a \vee b)=\phi(a) \wedge \phi(b)$.

If $\Sigma$ is self-dual, there is a one-to-one map $\phi$ of $\Sigma$ onto itself. If $a \leqq b$, then $\phi(a)=\phi(a \wedge b)=\phi(a) \vee \phi(b)$ which implies $\phi(b) \leqq \phi(a)$. Thus $\phi(0)=1$ and $\phi(1)=0$ and infraspaces map onto ultraspaces and conversely, so the number of infraspaces and ultraspaces must be equal.

In the lattice of topologies on a set $E$, if $|E|=n<\infty$, there are $n(n-1)$ ultraspaces (all principal) and $2^{n}-2$ infraspaces. If $|E| \geqq \aleph_{0}$, there are $2^{|E|}$ infraspaces and $2^{2^{|E|}}$ ultraspaces on $E$ (there are $2^{2|E|}$ ultrafiters on $E$ [2], [10]). Thus the number of ultraspaces equals the number of infraspaces only when $|E| \leqq 3$.

THEOREM 3.2. The lattice of topologies on $E$ is self-dual if and only if $|E| \leqq 3$.

Proof. If $|E|>3$, then $\Sigma$ cannot be self-dual by the preceding argument. If $|E|=1$ or $|E|=2, \Sigma$ is obviously self-dual. If $|E|=3$, there are twenty-nine topologies on $E$, but it can be seen by rotating the diagram on page 386 by $180^{\circ}$ that this lattice is also self-dual.

4. Topological properties. Ultraspaces may be studied easily because of their point-ultrafilter representation. In this section some topological properties of the ultraspaces are investigated and the maximal $T_{0}, T_{1}, T_{2}$, regular and normal topologies are characterized. Other topological properties of $T_{1}$-ultraspaces are considered in Gillman and Jerrison [10].

THEOREM 4.1. Every ultraspace is $T_{0}$, normal and extremally disconnected.

Proof. Let $\mathfrak{S}(x, \mathscr{U})$ be an ultraspace on $E$. Then $\mathfrak{S}(x, \mathscr{U})$ is a $T_{0}$-topology since for any two points of $E$ at least one as a set is open.

If $A$ and $B$ are disjoint closed sets, then $x \notin A$ or $x \notin B$. If $x \notin A$ then $A$ and $E-A$ are open and $\mathfrak{S}(x, \mathscr{U})$ is normal.

If $V$ is an open set and $x \in V$ then $V=\bar{V}$. If $x \notin V$ then either $E-V \in \mathscr{U}$ or $V \in \mathscr{U}$. If $E-V \in \mathscr{U}$ then $V=\bar{V}$. If $V \in \mathscr{U}$ then $\bar{V}=V \cup\{x\}$, but $V \cup\{x\} \in \mathscr{U}$ and hence is open. Thus $\subseteq(x, \mathscr{U})$ is extremally disconnected.

THEOREM 4.2. No ultraspace on a set $E$ is connected if $|E| \geqq 3$. 
Lattice of topologies on a three point set $E=\{a, b, c\}$

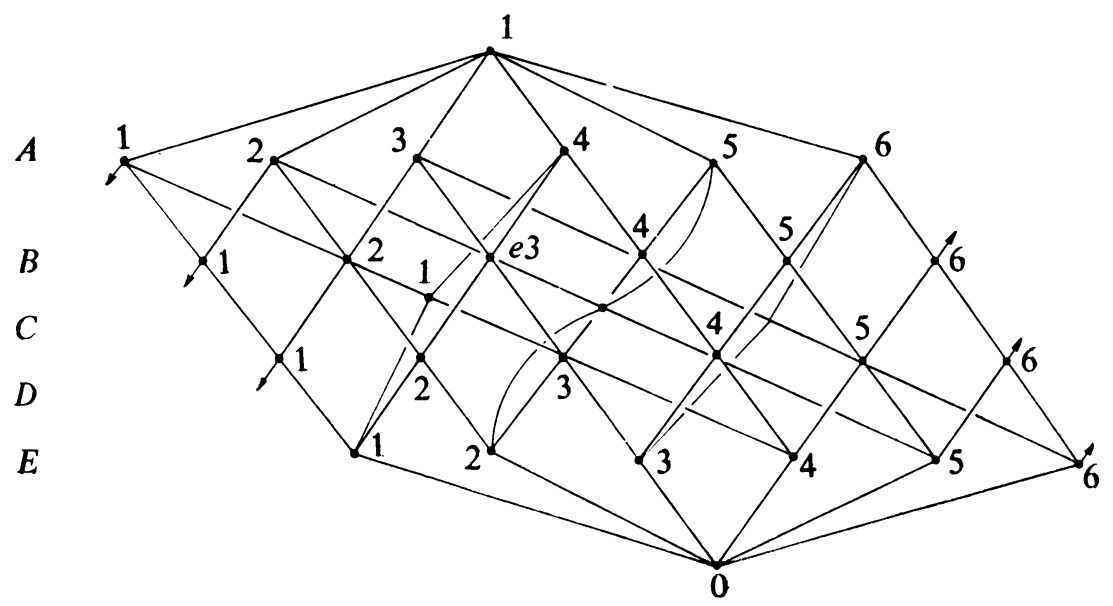

0 Trivial Topology

1 Discrete Topology

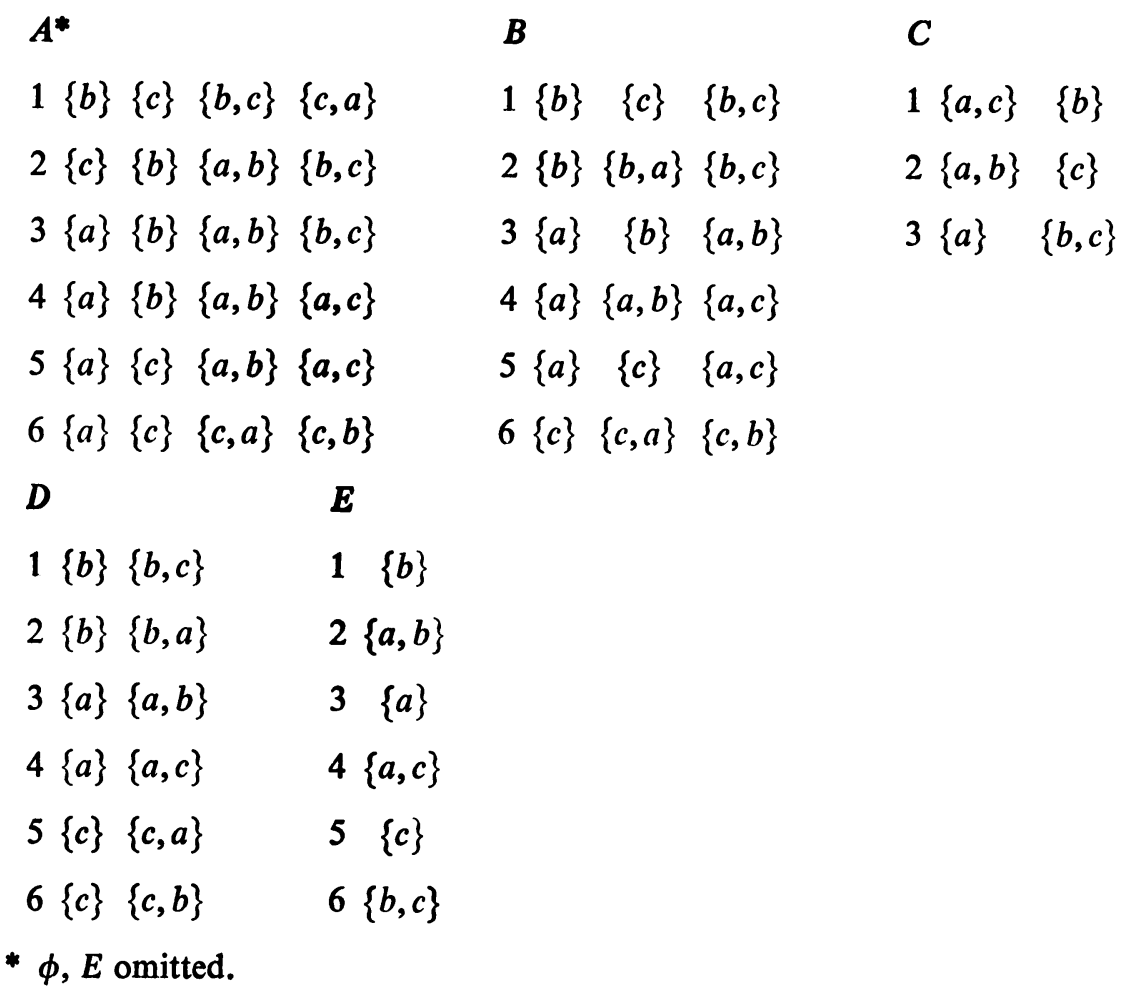


Proof. Let $\Im(p, \mathscr{V})$ be an ultraspace on $E$. If $|E| \geqq 3$, there is an $x \in E$ such that $x \neq p$ and $\mathscr{V} \neq \mathscr{U}(x)$. Thus $\{x\}$ and $E-\{x\}$ are both open.

An ultraspace is a $T_{1}$-topology if and only if it is a nonprincipal ultraspace (cf. Theorem 1.1).

THEOREM 4.3. An ultraspace is a $T_{2}$-topology if and only if is it a nonprincipal ultraspace.

Proof. For any two points, at least one as a set is open and its complement is also open and contains the other point.

A nonprincipal ultraspace is normal and $T_{1}$, hence is regular and completely regular. A principal ultraspace $\mathfrak{S}(x, \mathscr{U}(y))$ is not regular (since $\{x\}$ is a closed set, $y \notin\{x\}$, but every open set containing $x$ contains $y$ ), and therefore not completely regular. An ultraspace is totally disconnected (i.e. no connected subset contains more than one point) if and only if it is a nonprincipal ultraspace. Similarly, being nonprincipal is a necessary and sufficient condition for an ultraspace to be zero-dimensional (i.e. there is a base for the topology such that each set in the base is both open and closed).

A topological space is a door space if every subset is either open or closed. It is easy to see that every ultraspace is a door space. Actually it is not difficult to characterize door spaces.

THEOREM 4.4. A space is a door space if and only if the ultraspaces in its representation have either a common point or a common ultrafilter.

Proof. Let $\tau_{1}=\bigwedge\left\{\Im\left(x, \mathscr{U}_{i}\right) \mid i \in I_{1}\right\}$ and $\tau_{2}=\bigwedge\left\{\Im\left(y_{i}, \mathscr{V}\right) \mid i \in I_{2}\right\}$. A set not containing $x$ is in $\tau_{1}$ and if a set contains $x$, its complement is in $\tau_{1}$. Thus $\tau_{1}$ is a door space. Every set in $\mathscr{V}$ is in $\tau_{2}$ and if a set is not in $\mathscr{V}$, its complement is. Hence $\tau_{2}$ is a door space.

If $\tau \leqq \Im(x, \mathscr{U}) \wedge \Im(y, \mathscr{V})$ where $x \neq y$ and $\mathscr{U} \neq \mathscr{V}$, then there is a set $A$ such that $A \in \mathscr{U}$ and $E-A \in \mathscr{V}$. The set $(A \cup\{y\}) \cap(E-\{x\})$ is not in $\mathfrak{S}(y, \mathscr{V})$ and its complement is not in $\mathfrak{S}(x, \mathscr{U})$. Thus $(A \cup\{y\}) \cap(E-\{x\})$ is neither open nor closed in $\tau$.

Vaidyanathaswamy [17] erroneously stated that there are no maximal $T_{1}$-topologies. Liu [13] gave a necessary and sufficient condition for a topology to be a maximal $T_{1}$-topology and then proved that maximal $T_{1}$-topologies do exist by constructing an ultraspace with the aid of Zorn's lemma.

An immediate consequence of Theorem 1.1 is that every maximal $T_{1}$-topology is of the form $\mathfrak{S}(p, \mathscr{U})$ where $p \in E, \mathscr{U}$ is a nonprincipal ultrafilter on $E$, $\mathscr{U} \neq \mathscr{U}(p)$. Then every subset of $E-\{p\}$ and every set in $\mathscr{U}$ is open.

The maximal $T_{1}$-topologies are precisely the maximal $T_{2}$-topologies. Since every nondiscrete topology is coarser than some ultraspace and every ultraspace is a $T_{0}$-topology, the ultraspaces are the maximal $T_{0}$-topologies. Similarly, a normal topology is a maximal normal topology if and only if it is an ultraspace. 
All nonprincipal ultraspaces are maximal regular spaces, but there are also principal topologies which are regular.

A collection $\mathscr{D}$ of principal ultraspaces

$$
\left\{\Im\left(x_{1}, \mathscr{U}\left(x_{2}\right)\right), \mathfrak{S}\left(x_{2}, \mathscr{U}\left(x_{3}\right)\right), \cdots, \Im\left(x_{n}, \mathscr{I}\left(x_{1}\right)\right)\right\}
$$

is called a cycle of order $n$.

Lemma 4.5. A topology which is the intersection over a cycle of order 2 is a maximal regular topology.

Proof. Let $\tau=\mathfrak{S}(p, \mathscr{U}(q)) \wedge \mathfrak{S}(q, \mathscr{U}(p))$. Then $\tau$ is regular since every closed set is also open. The only nondiscrete topologies finer than $\tau$ are $\subseteq(p, \mathscr{T}(q))$ and $\mathfrak{S}(q, \mathscr{I}(p))$, neither of which is regular.

Lemma 4.6. A topology whose representation contains a cycle of order $n$ is coarser than a maximal regular topology of the form $\Im(x, \mathscr{l}(y)) \wedge \Im(y, \mathscr{l}(x))$.

Proof. Let $\mathscr{D}$ be a cycle of order $n$ in the representation of $\tau$. Then $\tau \leqq \wedge \mathscr{D}$ and since $\Im\left(x_{1}, \mathscr{l}\left(x_{2}\right)\right) \wedge \Im\left(x_{2}, \mathscr{U}\left(x_{3}\right)\right) \leqq \Im\left(x_{1}, \mathscr{U}\left(x_{3}\right)\right), \wedge \mathscr{D} \leqq \Xi\left(x_{1}, \mathscr{U}\left(x_{n}\right)\right) \wedge \Xi\left(x_{n}, \mathbb{U}\left(x_{1}\right)\right)$.

LemMa 4.7. A principal topology with no cycle in its representation is not regular.

Proof. By deleting $(x, x)$ from a relation $G$ corresponding to $\tau$, the topology $\tau_{G}$ remains the same. So assume $(x, x) \notin G$ for all $x \in E$.

Let $\tau=\bigwedge_{i \in I}\left\{\mathfrak{S}\left(x_{i}, \mathscr{U}_{i}\right)\right\}$ where $\left\{\mathfrak{S}\left(x_{i}, \mathscr{U}_{i}\right) \mid i \in I\right\}$ contains no cycle. If $B_{x_{0}}=\left\{y \in E \mid\right.$ there is a chain from $x_{0}$ to $y$ in $\left.G\right\}$ for some point $x_{0}$, then $x_{0} \notin B_{x_{0}}$ since for $x_{0} \in B_{x_{0}}$ and $\left(x_{0}, x_{0}\right) \notin G$, there is a cycle of order $n>1$ in $\left\{\mathfrak{S}\left(x_{i}, \mathscr{U}_{i}\right) \mid i \in I\right\}$ which is a contradiction. The set $B_{x_{0}}$ is open, so $E-B_{x_{0}}$ is closed. For $y \in B_{x_{0}}$ there is no open set containing $E-B_{x_{0}}$ which does not contain $y$ since $x_{0} \in E-B_{x_{0}}$. Thus $\tau$ is not regular.

THEOREM 4.8. A regular topology is a maximal regular topology if and only if it is a nonprincipal ultraspace or it is of the form $\Im(x, \mathscr{l}(y)) \wedge \Im(y, \mathscr{I l}(x))$ for some $x, y \in E$.

Proof. If $\tau$ is a nonprincipal ultraspace or $t \tau=\mathfrak{S}(x, \mathscr{U}(y)) \wedge \mathfrak{S}(y, \mathscr{U}(x))$, then $\tau$ is a maximal regular topology. Every $T_{1}$-topology and every mixed topology is coarser than some nonprincipal ultraspace and every regular principal topology is coarser than a maximal regular topology of the form

$$
\mathfrak{S}(x, \mathscr{U}(y) \wedge \mathfrak{S}(y, \mathscr{U}(x)) .
$$

5. Principal complements for principal topologies. In 1958, Hartmanis [11] proved that the lattice of topologies on a finite set is complemented. Since every topology on a finite set is a principal topology, this implies that the lattice $\Pi$ of principal topologies on a finite set is complemented. 
In this section it will be shown that the lattice $\mathscr{G}$ of preorder relations is a complemented lattice and hence, that the lattice $\Pi$ of principal topologies on an arbitrary set is complemented. Actually, this result is just a special case of the more general theorem concerning the complementation of $\Sigma$ and follows from Theorem 7.8.

A lattice complement of a preorder relation $G$ is a preorder relation $G^{\prime}$ such that $G \bigvee G^{\prime}=E \times E$ and $G \wedge G^{\prime}=\Delta=\{(x, x) \mid x \in E\}$.

The set complement $G^{*}=(E \times E)-G$ is not necessarily transitive and in general $G^{*} \wedge G \neq \Delta$.

THEOREM 5.1. The lattice $\mathscr{G}$ of preorder relations on a set $E$ is a complemented lattice.

Proof. Let $G$ be a preorder relation on a set $E$ and let $E=\bigcup_{\alpha \in \theta} E_{\alpha}$ where each $E_{\alpha}$ is a component of $E$ relative to $G$. Then for $x, y \in E, x$ and $y$ are in the same component if and only if there is a path from $x$ to $y$ in $G$, i.e., if $x G y$ or $y G x$.

Let $G_{1}=\{(y, x) \mid x G y$ and not $y G x\} \cup \Delta$. Select one point from each $E_{\alpha}$ and denote it by $x_{\alpha}$ (the $x_{\alpha}$ 's, $\alpha \in \theta$, will now remain fixed). Let $G_{2}=\left\{\left(x_{\alpha}, x_{\beta}\right) \mid \alpha, \beta \in \theta\right\} \cup \Delta$. The relations $G_{1}$ and $G_{2}$ are both preorder relations so $G^{\prime}=G_{1} \vee G_{2}$ is also a preorder relation.

If $x G y$, then $x$ and $y$ are in the same component $E_{\alpha}$ of $E$ and by definition, $(x, y) \notin G_{1}$, hence $(x, y) \notin G^{\prime}$. Therefore $G \wedge G^{\prime}=\Delta$.

For $(x, y) \in E \times E, x$ and $y$ are in components $E_{\alpha}$ and $E_{\beta}$, respectively, of $E$. If $\left(x, x_{\alpha}\right) \notin G$ then $\left(x, x_{\alpha}\right) \in G_{1}$ and if $\left(x_{\beta}, y\right) \notin G$ then $\left(x_{\beta}, y\right) \in G_{1}$. Since $\left(x_{\alpha}, x_{\beta}\right) \in G_{2}, \quad\left\{\left(x, x_{\alpha}\right),\left(x_{\alpha}, x_{\beta}\right),\left(x_{\beta}, y\right)\right\} \subset G \cup G^{\prime} \quad$ so $\quad(x, y) \in G \vee G^{\prime}$. Thus $G \vee G^{\prime}=E \times E$.

THEOREM 5.2. The lattice $\Pi$ of principal topologies is a complemented lattice.

This follows directly from Theorem 2.6 , since $\Pi$ and $\mathscr{G}$ are anti-isomorphic.

6. Reduction to $T_{1}$-topologies. Hartmanis [11], after concluding that the lattice of topologies on a finite set is complemented, posed the question: "Is the lattice of topologies on an infinite set complemented?" Gaifman [8] showed that this lattice is complemented if the set $E$ is countable. To obtain this result, he proved that if every $T_{1}$-topology on a set has a complement, then every topology on that set has one.

In this section it will be shown that if every $T_{1}$-topology on a set has a complement which is a principal topology, then every topology on that set has a principal complement. It will also be established that every $T_{1}$-topology on a countable set has a principal complement.

A topology $\tau$ is said to have a principal complement if there is a principal topology $\tau$ such that $\tau \vee \tau^{\prime}=1$ and $\tau \wedge \tau^{\prime}=0$. 
If $\tau$ is a topology on a set $E$ and $F \subset E$, then $\tau \mid F$ denotes the restriction of $\tau$ to $F$ (i.e., the relative topology on $F$ induced by $\tau$ ).

THEOREM 6.1. If every $T_{0}$-topology $\left(T_{1}\right.$-topology) $\tau$ on $E$ has a (principal) complement and $F \subset E$, then every $T_{0}$-topology $\left(T_{1}\right.$-topology) $\tau_{0}$ on $F$ has a (principal) complement.

Proof. Let $\tau_{0}$ be a $T_{0}$-topology on $F$, and define a topology $\tau$ on $E$ by $\tau=\left\{(A \subset E) \mid A \cap F \in \tau_{0}\right\}$. Then $\tau$ is a $T_{0}$-topology and hence has a complement $\tau^{\prime}$. For $x \in F$ there is a $U \in \tau, V \in \tau^{\prime}$ such that $\{x\}=U \cap V$. But $U \cap F \in \tau_{0}$ and $V \cap F \in \tau^{\prime} \mid F$ so $\{x\}=(U \cap F) \cap(V \cap F) \in \tau_{0} \vee \tau^{\prime} \mid F$. Thus $\tau_{0} \vee \tau^{\prime} \mid F=1$. For $U \in \tau_{0} \wedge \tau^{\prime} \mid F, U=V \cap F$ for some $V \in \tau^{\prime}$, hence $V \in \tau$ (by definition of $\tau$ ). Since $\tau \wedge \tau^{\prime}=0$, it follows that $V=\varnothing$ or $V=E$ and consequently that $U=\varnothing$ or $U=F$. Thus $\tau_{0} \wedge \tau^{\prime} \mid F=0$, and $\tau^{\prime} \mid F$ is a complement of $\tau_{0}$. If $\tau^{\prime}$ is principal, then for each $x \in F$ there is a minimal open set $B_{x} \in \tau^{\prime}$ containing $x$. Thus $B_{x} \cap F$ is a minimal open set in $\tau^{\prime} \mid F$ containing $x$, so $\tau^{\prime} \mid F$ is a principal topology.

THEOREM 6.2. If every $T_{0}$-topology on $E$ has a principal complement, then every topology on $E$ has a principal complement.

Proof. Gaifman [9] showed that if every $T_{0}$-topology on a set has a complement then every topology does. He did this in the following way:

Let $\tau$ be a topology on $E$. Define $x \approx y$ by: for every $V \in \tau, x \in V$ if and only if $y \in V$. Obviously $\approx$ is an equivalence relation. There exists a subset $E_{1}$ of $E$ whose intersection with every equivalence class consists of exactly one point. Now, $\tau_{1}=\tau \mid E_{1}$ is a $T_{0}$-topology and if every $T_{0}$-topology on $E$ has a complement then every $T_{0}$-topology on $E_{1}$ has a complement since $\left|E_{1}\right| \leqq|E|$. Denote this complement by $\tau_{1}^{\prime}$. Then $\tau^{\prime}=\left\{A \subset E \mid A \cap E_{1} \in \tau_{1}^{\prime}\right\}$ is a complement for $\tau$.

In Gaifman's notation, if every $T_{0}$-topology on $E$ has a principal complement then every $T_{0}$-topology on $E_{1}$ has a principal complement (Theorem 6.1). Thus, let $\tau_{1}^{\prime}$ be a principal complement of $\tau_{1}$. For each $x \in E-E_{1},\{x\} \in \tau^{\prime}$. For each $x \in E_{1}$, there is a minimal set in $\tau_{1}^{\prime}$ containing $x$ and this set is thus a minimal set in $\tau^{\prime}$ containing $x$. Therefore, $\tau^{\prime}$ is a principal topology (Theorem 2.3).

THEOREM 6.3. If every $T_{1}$-topology on a set $E$ has a principal complement, then every topology on $E$ has a principal complement.

Proof. By Theorem 6.2 it suffices to show that if every $T_{1}$-topology on $E$ has a principal complement, then every $T_{0}$-topology on $E$ has a principal complement. So let $\tau$ be a $T_{0}$-topology on $E$.

Under the assumption that every $T_{1}$-topology on $E$ has a complement, Gaifman [9] deduced that every $T_{0}$-topology on $E$ has a complement in the following way: 
He defined an ordered family of disjoint sets $E_{\alpha}$, such that $\tau_{\alpha}=\tau \mid E_{\alpha}$ is a $T_{1}$-topology (the other properties of the $E_{\alpha}$ are not essential to this proof), and constructed the topology $\tau^{\prime}$ in the following way:

Since each $\tau_{\alpha}$ is a $T_{1}$-topology on $E_{\alpha}$, by Theorem $6.1 \tau_{\alpha}$ has a complement $\tau_{\alpha}^{\prime}$.

The topology $\tau^{\prime}$ is generated by sets of the form:

(i) $\{x\}$, where $x \notin \bigcup_{\alpha} E_{\alpha}$.

(ii) $V, V \in \tau_{\alpha}^{\prime}$ where $\alpha$ is such that for every $\beta>\alpha$ and for every $W \in \tau$ such that $E_{\alpha} \subseteq W, W \cap\left[\bigcup_{\beta \subseteq v} E_{v}\right] \neq \varnothing$.

(iii) $V \cup \bigcup_{\beta \leqq v} E_{v}$ where $V \in \tau_{\alpha}^{\prime}$ and $\alpha, \beta$ are such that $\beta>\alpha$ and for some $W \in \tau, E_{\alpha} \subseteq W$, and $W \cap\left[\bigcup_{\beta \leqq v} E_{v}\right]=\varnothing$.

Gaifman proved $\tau \vee \tau^{\prime}=1$ and $\tau \wedge \tau^{\prime}=0$.

Now, if $\tau^{*}$ is defined in a modified form as the topology generated by the following sets:

(i) and (ii) as above,

(iii) $V \cup \bigcup_{\beta . \leqq v} E_{v}$ where $V \in \tau_{\alpha}^{\prime}$ and $\beta_{\alpha}, \alpha$ are such that $\beta_{\alpha}>\alpha$ ( $\beta_{\alpha}$ fixed for each $\alpha$ ) and for some $W \in \tau, E_{\alpha} \subseteq W$, and $W \cap\left[\bigcup_{\beta_{\alpha} \leqq v} E_{v}\right]=\varnothing$, then it can be shown that $\tau^{*}$ is a principal topology if each $\tau_{\alpha}^{\prime}$ is. First, $\tau^{*}$ is a union of sets of the form (i), (ii) and (iii). The intersection of sets of the form (i) with different sets of the form (i), (ii) or (iii) is empty. The intersection of sets of the form (ii) with sets of the form (ii) or (iii) is of the form (ii) and the intersection of two sets of the form (iii) is again of form (iii). To elaborate this last point let $U_{i}=V_{i} \cup \bigcup_{\beta \alpha_{i} \leqq \nu} E_{v}, i=1,2$, be two sets of form (iii) where $V_{i} \in \tau_{\alpha_{i}}, i=1,2$. If $\alpha_{1}=\alpha_{2}$ then $\beta_{\alpha_{1}}=\beta_{\alpha_{2}}$ and $U_{1} \cap U_{2}=\left(V_{1} \cap V_{2}\right) \cup \bigcup_{\beta_{\alpha_{1}} \leqq v} E_{v}$ and this is of form (iii). If $\alpha_{1}<\alpha_{2}$ then $U_{1} \cap U_{2}=U_{2}$ if $\beta_{\alpha_{1}} \leqq \alpha_{2}$, and $U_{1} \cap U_{2}=\bigcup_{\beta_{3} \leqq v} E_{v}$ if $\alpha_{2}<\beta_{\alpha_{1}}$, where $\beta_{3}=\max \left(\beta_{\alpha_{1}}, \beta_{\alpha_{2}}\right)$. This last set is of the form (iii) also, where $V=\varnothing$.

Second, $\tau \bigvee \tau^{*}=1$. If $x \notin \bigcup_{\alpha} E_{\alpha}$, then $\{x\} \in \tau^{*}$. If $x \in E_{\alpha}$, then $\{x\}=V \cap V^{\prime}$ where $V \in \tau_{\alpha}^{\prime}, V^{\prime} \in \tau_{\alpha}^{\prime}$ and $V=U \cap E_{\alpha}$ for some $U \in \tau$. If, for every $W \in \tau$ and every $\beta>\alpha, \quad E_{\alpha} \subseteq W$ implies $W \cap\left[\bigcup_{\beta \leqq v} E_{v}\right] \neq \varnothing$ then $V^{\prime} \in \tau^{*}$ so $\{x\}=\left(U \cap E_{\alpha}\right) \cap V^{\prime}=U \cap V^{\prime} \in \tau \vee \tau^{*}$. Otherwise there is a $\beta_{\alpha}>\alpha$ and some $W \in \tau$ such that $E_{\alpha} \subseteq W$ and $W \cap\left[\bigcup_{\beta_{x} \leqq v} E_{v}\right]=\varnothing$. Then $V^{\prime \prime}=V^{\prime} \cup \bigcup_{\beta_{x} \leqq v} E_{v} \in \tau^{*}$ and $(U \cap W) \cap V^{\prime \prime}=\{x\}$. Hence $\tau \vee \tau^{*}=1$. Since $\tau^{*} \subseteq \tau^{\prime}, \tau \wedge \tau^{\prime}=0$ implies $\tau \wedge \tau^{*}=0$. Thus $\tau^{*}$ is a complement of $\tau$.

Since $\tau_{\alpha}$ is a $T_{1}$-topology, by Theorem 6.1, $\tau_{\alpha}^{\prime}$ is a principal topology for each $\alpha$.

For $x \notin \bigcup_{\alpha} E_{\alpha}$, there is a minimal open set containing $x$ in $\tau^{*}$, namely $\{x\}$.

For $x \in E_{\alpha}$, there is a minimal open set $B_{x} \in \tau_{\alpha}^{\prime}$ containing $x$. In case (ii) $B_{x} \in \tau^{*}$. Any set of form (ii) in $\tau^{*}$ containing $x$ must be in $\tau_{\alpha}^{\prime}$ and hence contains $B_{x}$. Any set of form (iii) in $\tau^{*}$ containing $x$, contains $E_{\alpha}$, hence $B_{x}$. So $B_{x}$ is a minimal open set in $\tau^{*}$ containing $x$. In case (iii), $V_{x}=B_{x} \cup \bigcup_{\beta_{x} \leqq \nu} E_{v} \in \tau^{*}$. Let $W \in \tau^{*}$ such that $x \in W . W$ is the union of sets of the form (i), (ii) and (iii) but only the part of form (iii) can contain $x$. So we assume $W=U \cup \bigcup_{\beta_{\alpha_{1}} \leqq \gamma} E_{\nu}$ where $U \in \tau_{\alpha_{1}}^{\prime}$. 
If $\alpha_{1}=\alpha$, then $B_{x} \subset U$ so $V_{x} \subset W$. If $\alpha_{1}<\alpha$ and $\beta_{\alpha_{1}} \leqq \alpha$ then $W \cap V_{x}=V_{x}$ so $V_{x} \subset W$. If $\alpha_{1}<\alpha<\beta_{\alpha_{1}}$ then $x \notin W$ and if $\alpha<\alpha_{1}$ then $x \notin W$. So $V_{x}$ is a minimal open set in $\tau^{*}$ containing $x$. Thus $\tau^{*}$ has a base of open sets, minimal at each point and $\tau^{*}$ is a principal topology (Theorem 2.3).

THEOREM 6.4. A T T -topology on a countable set has a principal complement.

Proof. Let $\tau$ be a $T_{1}$-topology on a countable set $E$. Let $E$ be ordered as $\left\{x_{i} \mid i=1,2, \cdots\right\}$ where $\left\{x_{1}\right\} \notin \tau$. If $\left\{x_{i}\right\} \in \tau, i=1,2, \cdots, \tau=1$ and has a principal complement, 0 . Let $G$ be a relation on $E$ defined as $G=\left\{\left(x_{i}, x_{j}\right) \in E \times E \mid j<i\right\}$. Then the topology $\tau^{\prime}$ defined by $G$ is a principal topology and is a complement of $\tau$.

For each $x_{i} \in E, i \neq 1$, the set $B_{x_{i}}=\left\{x_{j} \in E \mid j \leqq i\right\}$ is open in $\tau^{\prime}$. Since $B_{x_{i}}$ is finite, $U=E-\left\{B_{x_{i}}-\left\{x_{i}\right\}\right\}$ is an open set in $\tau$ containing $x_{i}$. Thus $\left\{x_{i}\right\}=B_{x_{i}} \cap U \in \tau^{\prime} \vee \tau$. And since $\left\{x_{1}\right\} \in \tau^{\prime}, \tau \vee \tau^{\prime}=1$.

Let $U \in \tau \wedge \tau^{\prime}, U \neq \varnothing$. If $x_{i} \in U$, then $B_{x_{i}} \subset U$ since $U \in \tau^{\prime}$. Hence $x_{1} \in U$ and since $\tau$ is a $T_{1}$-topology, $U$ must be infinite. For every $x_{k} \in E$ there is a $j \geqq k$ such that $x_{j} \in U$. But $B_{x_{j}} \subset U$ implies $x_{k} \in U$. Thus $U=E$ and $\tau \wedge \tau^{\prime}=0$.

THEOREM 6.5. Every topology on a countable set has a principal complement.

7. The general theorem. In this section it will be shown that every topology has a principal lattice complement. Several preliminary theorems are proved first.

THEOREM 7.1. Let $\tau$ be a topology on a set $E$, where $E=E_{1} \cup E_{2}$ and $E_{1} \cap E_{2}=\varnothing$ such that $\tau_{1}=\tau \mid E_{1}$ and $\tau_{2}=\tau \mid E_{2}$ have (principal) lattice complements $\tau_{1}$ and $\tau_{2}^{\prime}$ respectively. Then $\tau$ has a (principal) complement.

Proof. If either $E_{1}$ or $E_{2}$ is empty, the theorem is trivial. So suppose both are nonempty.

Case (a). Suppose $E_{1}$ and $E_{2}$ are open sets in $\tau$. Let $x_{1} \in E_{1}$ and $x_{2} \in E_{2}$. Let $\tau^{\prime}$ be the topology generated by sets of the form:

(i) $U$, where $U \in \tau_{1}^{\prime}$ and $x_{1} \notin U$,

(ii) $V$, where $V \in \tau_{2}^{\prime}$ and $x_{2} \notin V$,

(iii) $U \cup V$, where $x_{1} \in U \in \tau_{1}^{\prime}$ and $x_{2} \in V \in \tau_{2}^{\prime}$.

(If $\tau_{1}^{\prime}$ and $\tau_{2}^{\prime}$ are principal topologies, then $\tau^{\prime}$ is the principal topology defined by the relation $G=G_{1} \cup G_{2} \cup\left\{\left(x_{1}, x_{2}\right),\left(x_{2}, x_{1}\right)\right\}$ where $G_{1}$ defines $\tau_{1}^{\prime}$ and $G_{2}$ defines $\tau_{2}^{\prime}$.)

It is not difficult to see that the restriction of an open set in $\tau^{\prime}$ to $E_{1}$ (or $E_{2}$ ) is open in $\tau_{1}^{\prime}$ (or $\tau_{2}^{\prime}$ ).

For $x \in E$, suppose $x \in E_{1}$. Then there are open sets $U \in \tau_{1}$, and $U^{\prime} \in \tau_{1}^{\prime}$ such that $\{x\}=U \cap U^{\prime}$. Since $E_{1}$ is open, $U \in \tau$, and either $U^{\prime} \in \tau^{\prime}$, if $x_{1} \notin U^{\prime}$, or $U^{\prime} \cup V^{\prime} \in \tau^{\prime}$ if $x_{1} \in U^{\prime}$ where $x_{2} \in V^{\prime} \in \tau_{2}^{\prime}$. In either case, $\{x\} \in \tau \vee \tau^{\prime}$ for each $x \in E_{1}$. Similarly $\{x\} \in \tau \vee \tau^{\prime}$ for each $x \in E_{2}$, so $\tau \vee \tau^{\prime}=1$. 
Let $A \in \tau \wedge \tau^{\prime}, A \neq \varnothing$. Thus $A \cap E_{1} \in \tau_{1} \wedge \tau_{1}^{\prime}$ and $A \cap E_{2} \in \tau_{2} \wedge \tau_{2}^{\prime}$. Some $x$ is in $A$, and if $x \in E_{1}$, then $A \cap E_{1}=E_{1}$. But since $x_{1} \in A$ and $A \in \tau^{\prime}, x_{2} \in A$. Thus $A \cap E_{2}=E_{2}$ and $A=E$. Hence $\tau \wedge \tau^{\prime}=0$.

Case (b). Suppose neither $E_{1}$ nor $E_{2}$ is open. Let $\tau^{\prime}$ be the topology generated by sets of the form:

(i) $U$, where $U \in \tau_{1}^{\prime}$,

(ii) $V$, where $V \in \tau_{2}^{\prime}$.

(If $\tau_{1}^{\prime}$ and $\tau_{2}^{\prime}$ are principal topologies, then $\tau^{\prime}$ is the principal topology defined by the relation $G=G_{1} \cup G_{2}$ where $G_{1}$ defines $\tau_{1}^{\prime}$ and $G_{2}$ defines $\tau_{2}^{\prime}$.)

For $x \in E$, if $x \in E_{1}$ then there is a $U \in \tau_{1}$ and $U^{\prime} \in \tau_{1}^{\prime}$ such that $\{x\}=U \cap U^{\prime}$. But $U=V \cap E_{1}$ where $V \in \tau$. Hence $\{x\}=V \cap E_{1} \cap U^{\prime}=V \cap U^{\prime} \in \tau \vee \tau^{\prime}$ since $U^{\prime} \in \tau^{\prime}$. Similarly, if $x \in E_{2},\{x\} \in \tau \vee \tau^{\prime}$ so $\tau \vee \tau^{\prime}=1$.

Let $A \in \tau \wedge \tau^{\prime}, A \neq \varnothing$. If $x \in E_{1} \cap A, A \cap E_{1} \in \tau_{1} \wedge \tau_{1}^{\prime}$ implies $A \cap E_{1}=E_{1}$. But $E_{1} \notin \tau$ so some $x \in E_{2}$ is in $A$. Thus $A \cap E_{2}=E_{2}$ and $A=E$. Similarly if $A \cap E_{2} \neq \varnothing$, then $A=E$. Therefore $\tau \wedge \tau^{\prime}=0$.

Case (c). Suppose $E_{1}$ is open but $E_{2}$ is not open. Let $x_{1} \in E_{1}$ and $x_{2} \in E_{2}$ and let $\tau^{\prime}$ be the topology generated by sets of the form:

(i) $U$, where $U \in \tau_{1}^{\prime}$ and $x_{1} \notin U$,

(ii) $V$, where $V \in \tau_{2}^{\prime}$,

(iii) $U \cup V$, where $x_{1} \in U \in \tau_{1}^{\prime}$ and $x_{2} \in V \in \tau_{2}^{\prime}$. (If $\tau_{1}^{\prime}$ and $\tau_{2}^{\prime}$ are principal topologies, then $\tau^{\prime}$ is the principal topology defined by the relation $G=G_{1} \cup G_{2} \cup\left\{\left(x_{1}, x_{2}\right)\right\}$, where $G_{1}$ defines $\tau_{1}^{\prime}$ and $G_{2}$ defines $\tau_{2}^{\prime}$.)

The restriction of an open set in $\tau^{\prime}$ to $E_{1}$ (or $E_{2}$ ) is open in $\tau_{1}^{\prime}$ (or $\tau_{2}^{\prime}$ ).

For $x \in E_{1},\{x\}=U \cap U^{\prime}$ where $U \in \tau_{1}$ and $U^{\prime} \in \tau_{1}^{\prime}$. But $U \in \tau$ and if $x_{1} \notin U^{\prime}$ then $U^{\prime} \in \tau^{\prime}$ and if $x_{1} \in U^{\prime}$ then $U^{\prime} \cup V^{\prime} \in \tau^{\prime}$ where $x_{2} \in V^{\prime} \in \tau_{2}^{\prime}$. In either case, $\{x\} \in \tau \vee \tau^{\prime}$. For $x \in E_{2},\{x\}=U \cap U^{\prime}$ where $U \in \tau_{2}$ and $U^{\prime} \in \tau_{2}$. But $U^{\prime} \in \tau^{\prime}$ and $U=V \cap E_{2}$ where $V \in \tau$. Thus $\{x\}=V \cap U^{\prime}$, hence $\{x\} \in \tau \vee \tau^{\prime}$. Therefore $\tau \vee \tau^{\prime}=1$.

Let $A \in \tau \wedge \tau^{\prime}, \quad A \neq \varnothing$. If $A \cap E_{1} \neq \varnothing$, then $A \cap E_{1}=E_{1}$ since $A \cap E_{1} \in \tau_{1} \wedge \tau_{1}^{\prime}$. But $x_{1} \in E_{1}$ implies $x_{2} \in A$ since $A \in \tau^{\prime}$ and thus $A \cap E_{2}=E_{2}$. If $A \cap E_{2} \neq \varnothing$, then $A \cap E_{2}=E_{2}$ since $A \cap E_{2} \in \tau_{2} \wedge \tau_{2}^{\prime}$. But $E_{2} \notin \tau$ so some $x \in E_{1}$ must be in $A$. Hence $A \cap E_{1}=E_{1}$ and $A=E$. Therefore $\tau \wedge \tau^{\prime}=0$.

THEOREM 7.2. If $\tau$ is a topology on $E$ and $E=I \cup(E-I)$, where $I$ is the set of isolated points of $\tau$, and if $\tau \mid(E-I)$ has a (principal) lattice complement, then $\tau$ has a (principal) lattice complement.

This is an immediate consequence of Theorem 7.1, since $\tau \mid I$ is the discrete topology and as such has a principal complement.

THEOREM 7.3. If every topology ( $T_{1}$-topology) with no isolated points has a principal complement, then every topology $\left(T_{1}\right.$-topology) has a principal complement. 
Proof. Let $\tau$ be a topology $\left(T_{1}\right.$-topology) on a set $E$ and let $I=I_{1}$ be the set of isolated points of $\tau$. It can be assumed that $I_{1} \neq \varnothing$. Let $I_{2}$ be the set of isolated points of $\tau \mid\left(E-I_{1}\right)$.

Let $\lambda$ be an ordinal number and suppose $I_{\lambda}$ has been defined for all $\lambda<v$. Define $I_{v}$ as the set of isolated points of $\tau \mid\left(E-\bigcup_{\lambda<v} I_{\lambda}\right)$.

Thus a family of disjoint sets $\left\{I_{\lambda} \mid \lambda<\mu\right\}$ is inductively defined where $|E|<|\mu|$ so that, for each $v<\mu, I_{v}$ is the set of isolated points of $\tau \mid\left(E-\bigcup_{\lambda<v} I_{\lambda}\right)$.

There is some $\gamma<\mu$ such that $I_{\gamma}=\varnothing$. Let $\gamma$ be the first ordinal such that $I_{\gamma}=\varnothing$ and let $E_{1}=\bigcup_{\lambda<\gamma} I_{\lambda}$ and $E_{2}=E-E_{1}$. Since $I_{\gamma}=\varnothing$, the topology $\tau \mid E_{2}$ has no isolated points. Let $\tau_{1}=\tau \mid E_{1}$.

Select $x_{\lambda} \in I_{\lambda}$ for each $\lambda<\gamma$. Let

$$
G=\bigcup_{\lambda<\gamma}\left(I_{\lambda} \times I_{\lambda}\right) \cup\left\{\left(x_{\lambda}, x_{v}\right) \mid \lambda<v<\gamma\right\} .
$$

Then the principal topology $\tau^{\prime}$ on $E_{1}$ defined by $G$ is a complement of $\tau_{1}$.

Let $V \in \tau_{1} \wedge \tau^{\prime}, V \neq \varnothing$. If $V \cap I_{\lambda} \neq \varnothing, \lambda<v$, then $I_{\lambda} \subset V$ since $V \in \tau^{\prime}$. It follows that $x_{\lambda} \in V$, hence $x_{v} \in V$ and $I_{v} \subset V$, for $\lambda<v<\gamma$. Thus $V=\bigcup_{\delta \leqq \lambda<\gamma} I_{\lambda}$ for the least ordinal $\delta$ such that $V \cap I_{\delta} \neq \varnothing$.

Since $V \in \tau_{1}, \quad V=E_{1} \cap V^{\prime}$ for some $V^{\prime} \in \tau$. As $x_{\delta} \in V$ is isolated in $\tau \mid\left(E-\bigcup_{\lambda<\delta} I_{\lambda}\right),\left\{x_{\delta}\right\}=W \cap\left(E-\bigcup_{\lambda<\delta} I_{\lambda}\right)$ for some $W \in \tau$. But $V \subset V^{\prime} \subset E$ - $\bigcup_{\lambda<\delta} I_{\lambda}$, and hence $W \cap V^{\prime}=\left\{x_{\delta}\right\}$ is isolated in $\tau$. Thus $\delta=1$ and $V=E_{1}$. Therefore $\tau_{1} \vee \tau^{\prime}=0$.

For each $x \in E_{1}, x$ is in some $I_{v}$. Thus $x$ is an isolated point in $\tau \mid\left(E-\bigcup_{\lambda<v} I_{\lambda}\right)$. There is then a $W \in \tau$ such that $\{x\}=W \cap\left(E-\bigcup_{\lambda<v} I_{\lambda}\right)$, but $\left\{E-\bigcup_{\lambda<\nu} I_{\lambda}\right\} \in \tau^{\prime}$ and $W \cap E_{1} \in \tau_{1}$. Therefore $\tau_{1} \vee \tau^{\prime}=1$.

It has been shown that $\tau \mid E_{1}$ has a principal complement $\tau^{\prime}$. If $E_{2}=\varnothing$ then $\tau^{\prime}$ is a principal complement of $\tau$. If $E_{2} \neq \varnothing$, then $\tau \mid E_{2}$ has no isolated points, and hence by assumption a principal complement. (If $\tau$ is a $T_{1}$-topology, so is $\tau \mid E_{2}$.) But then by Theorem 7.1, $\tau$ has a principal complement.

The next theorem in this section is an extension of the following result of Berri [3], [4]: A topology on a set $E$ has a complement if there is a decomposition of $E$ into countable sets such that no union of any proper subcollection is open.

THEOREM 7.4. Let $\tau$ be a topology on a set $E$ such that

(i) $E=\bigcup_{\alpha \in \theta} E_{\alpha}$, where the $E_{\alpha}$ 's are pairwise disjoint,

(ii) $\tau_{\alpha}=\tau \mid E_{\alpha}$ has a (principal) lattice complement $\tau_{\alpha}^{\prime}$, and

(iii) for all $V \in \tau$, if $V \neq E, \varnothing$, then $V$ is not the union of $E_{\alpha}$ 's .

Then $\tau$ has a (principal) complement $\tau^{\prime}$. If some $\tau_{\alpha}^{\prime}$ has an isolated point, so does $\tau^{\prime}$.

Proof. Let $\tau^{\prime}$ consist of all sets of the form $\bigcup_{\alpha \in \theta} V_{\alpha}$ where $V_{\alpha} \in \tau_{\alpha}^{\prime}$ for all $\alpha \in \theta$. (If each $\tau_{\alpha}^{\prime}$ is princinal, $\tau^{\prime}$ is the principal topology defined by the union of the relations representing the $\tau_{\alpha}^{\prime}$.) 
Let $x \in E$. Then $x \in E_{\alpha}$ for some $\alpha \in 0$. There is a $V \in \tau_{\alpha}$ and a $V^{\prime} \in \tau_{\alpha}^{\prime}$ such that $\{x\}=V \cap V^{\prime}$. Since $V^{\prime} \in \tau^{\prime}$ and $V=U \cap E_{\alpha}$ for some $U \in \tau,\{x\}=\left(U \cap E_{\alpha}\right) \cap V^{\prime}$ $=U \cap V^{\prime} \in \tau \vee \tau^{\prime}$. Thus $\tau \vee \tau^{\prime}=1$.

If $V \in \tau \wedge \tau^{\prime}$ then $V \cap E_{\alpha} \in \tau_{\alpha} \wedge \tau_{\alpha}^{\prime}$, hence for all $\alpha \in \theta$, either $V \cap E_{\alpha}=E_{\alpha}$ or $V \cap E_{\alpha}=\varnothing$. If $V \cap E_{\alpha}=\varnothing$ for all $\alpha \in \theta$, then $V=\varnothing$. If $V \cap E_{\alpha}=E_{\alpha}$ for some $\alpha$, then $V \cap E_{\alpha}=E_{\alpha}$ for all $\alpha \in \theta$ by hypothesis, and $V=E$. Therefore $\tau \wedge \tau^{\prime}=0$. If $\{x\} \in \tau_{\alpha}^{\prime}$ for some $\alpha$, then $\{x\} \in \tau^{\prime}$.

THEOREM 7.5. Let $\tau$ be a $T_{1}$-topology on a set E containing a proper open set $S$ with at least two points, such that $\tau_{S}=\tau \mid S$ has a (principal) complement $\tau_{S}^{\prime}$ with an isolated point. Then $\tau$ has a (principal) complement with an isolated point.

Proof. Let $x_{0} \in S$ be an isolated point, i.e., $\left\{x_{0}\right\} \in \tau_{S}^{\prime}$, and let $y_{0} \in S, y_{0} \neq x_{0}$. Define $\tau^{\prime}$ as unions of sets of the form:

(i) $V$, where $V \in \tau_{S}^{\prime}$ and $y_{0} \notin V$,

(ii) $V \cup(E-S) \cup\left\{x_{0}\right\}$, where $y_{0} \in V \in \tau_{S}^{\prime}$,

(iii) $\{z\} \cup\left\{x_{0}\right\}$, for each $z \in E-S$.

The intersection of sets of form (i) with sets of the form (i), (ii) or (iii) is again a set of form (i). A set of form (ii) intersected with a set of form (ii) is a set of form (ii) and intersected with a set of form (iii) is of form (iii). The intersection of two distinct sets of form (iii) is a set of form (i). Thus these sets form a base for a topology.

(If $\tau_{s}^{\prime}$ is a principal topology with defining relation $G_{S}$, then $\tau^{\prime}$ is the principal topology defined by

$$
G=G_{S} \cup\left\{y_{0}\right\} \times(E-S) \cup(E-S) \times\left\{x_{0}\right\} .
$$

It must be shown that $\tau \vee \tau^{\prime}=1$ and $\tau \wedge \tau^{\prime}=0$. Let $x \in E$. If $x \in E-S$, then $\{x\}=\left\{x, x_{0}\right\} \cap\left(E-\left\{x_{0}\right\}\right) \in \tau^{\prime} \vee \tau$. If $x \in S$ and $x \neq y_{0}$, then $\{x\}=U \cap V$ where $U \in \tau_{S}$ and $V \in \tau_{S}^{\prime}$. If $y_{0} \notin V$ then $V \in \tau^{\prime}$ and since $U \in \tau,\{x\} \in \tau \vee \tau^{\prime}$. If $y_{0} \in V$ then $V^{*}=V \cup(E-S) \cup\left\{x_{0}\right\} \in \tau^{\prime} \quad$ and $U^{*}=U \cap\left(E-\left\{x_{0}\right\}\right) \in \tau$. Thus $\{x\}=U^{*} \cap V^{*} \in \tau \vee \tau^{\prime}$. If $x=y_{0}$, then $\left\{y_{0}\right\}=V \cap U$ where $V \in \tau_{s}^{\prime}$ and $U \in \tau_{s}$. Thus $\left\{y_{0}\right\}=\left(V \cup(E-S) \cup\left\{x_{0}\right\}\right) \cap\left(U \cap\left(E-\left\{x_{0}\right\}\right)\right) \in \tau^{\prime} \vee \tau$. Therefore $\tau \vee \tau^{\prime}=1$. Let $A \in \tau \wedge \tau^{\prime}$. Since $A \cap S \in \tau_{S} \cap \tau_{S}^{\prime}, A \cap S=S$ or $A \cap S=\varnothing$. If $A \cap S=S$, then since $y_{0} \in S \in \tau_{S}^{\prime}, A$ must contain $E-S$ and hence $A=E$. If $A \cap S=\varnothing$, then $A=\varnothing$, since $z \in A \cap(E-S)$ would imply $x_{0} \in A \cap S$. Hence $\tau \wedge \tau^{\prime}=0$.

Since $\left\{x_{0}\right\} \in \tau_{s}^{\prime},\left\{x_{0}\right\} \in \tau^{\prime}$. Thus $\tau^{\prime}$ has an isolated point.

THEOREM 7.6. If $\tau$ is a $T_{1}$-topology on a set $E$ such that

(i) $E$ is ordered as $\left\{x_{\alpha}\right\}_{\alpha<\mu}$ where $\mu$ is the smallest ordinal of cardinality $|E|$, 
(ii) each nonempty open set in $\tau$ has the same cardinality as $E$,

(iii) for each $x \in E$ there is an open set containing $x$ which does not contain any predecessors of $x$, then $\tau$ has a principal complement with a insolated point.

Proof. Let $\tau^{\prime}$ be the principal topology defined by the relation $G=\{(x, y) \mid y<x\}$.

Let $U \in \tau \wedge \tau^{\prime}, U \neq \varnothing$. For each $x \in E$ there is a $z \in E . z>x$, such that $z \in U$. Otherwise it follows from the well-order on $E$ that $|U|<|E|$. Thus $x \in U$ since $U \in \tau^{\prime}$ and $(z, x) \in G$, and $U=E$. Hence $\tau \wedge \tau^{\prime}=0$.

For each $x \in E$, the set $V=\{y \mid y \leqq x\} \in \tau^{\prime}$ and there is a set $U \in \tau$ which contains $x$ but contains no predecessors of $x$. Thus $\{x\}=U \cap V \in \tau \vee \tau^{\prime}$ and $\tau \bigvee \tau^{\prime}=1$.

For the first element $x_{1}$ in the well-order, $\left\{x_{1}\right\} \in \tau^{\prime}$.

THEOREM 7.7. A $T_{1}$-topology with no isolated points has a principal complement with an isolated point.

Proof. If there is some $T_{1}$-topology with no isolated points which does not have a principal complement with an isolated point, then there is a first cardinal $\aleph$ such that some $T_{1}$-topology $\tau$ on $E$, where $\tau$ has no isolated points' and $|E|=\aleph$, fails to have a principal complement with an isolated point.

No set in $\tau$ has isolated points in its relative topology, hence by Theorem 7.5 every nonempty set in $\tau$ must have cardinality $\aleph$.

Let $E$ be well ordered as $\left\{x_{\alpha}\right\}_{\alpha<\mu}$ where $\mu$ is the smallest ordinal of cardinality $\aleph$.

(a) If for each $x \in E$ there is an open set containing $x$ which contains no predecessors of $x$, then $\tau$ has a principal complement with an isolated point by Theorem 7.6.

Hence, the set $S=\{x \in E \mid$ every open set containing $x$ contains predecessors of $x\}$ is not empty. No nonempty subset of $S$ can be in $\tau$ since it would be an open set not containing the predecessors of its first element. Since $S \notin \tau, E-S \neq \varnothing$.

(b) Suppose there is a nonempty set $U \in \tau$ such that $U \cap S=\varnothing$. Then $\tau_{U}=\tau \mid U$ satisfies the conditions of Theorem 7.6, and by Theorem 7.6 and Theorem 7.5, $\tau$ has a principal complement with an isolated point.

(c) Thus $S$ must be dense in $\tau$. Also, since no nonempty subset of $S$ is open, $E-S$ is also dense in $\tau$.

Let us say that a subset $M \subset E$ with the induced well-order has property $P$ if for every $x \in M$ there is an open set containing $x$ which does not contain any predecessors of $x$ in $M$.

Consider sets of disjoint, dense subsets of $E$ having property $P$ and let $\Gamma$ be the collection of all such sets. Since $E-S$ is dense and has property $P, \Gamma$ is not empty. The union of a chain of sets of disjoint dense subsets of $E$ having property $P$ is again such a set. Thus, by Zorn's Lemma, $\Gamma$ has a maximal element $\mathfrak{M}=\left\{W_{i} \mid i \in \theta\right\}$. 
Denote by $F$ the set $E-\bigcup_{i \in \theta} W_{i}, W_{i} \in \mathfrak{M}$, and let $\tau_{F}=\tau \mid F$. The well-order on $E$ induces a well-order on $F$. Let $T=\left\{x \in F \mid\right.$ every set in $\tau_{F}$ containing $x$ contains predecessors of $x$ in $F\}$. Then $F=T \cup(F-T)$ where $F-T$ has property $P$ with respect to $\tau_{F}$. No subset of $T$ is in $\tau_{F}$ so $F-T$ is dense in $\tau_{F}$. If $F$ is dense in $\tau$, since $F-T$ is dense in $\tau_{F}, F-T$ is dense in $\tau$, which contradicts the maximality of $\mathfrak{M}$.

(d) Thus $F$ is not dense in $\tau$ and there is a set $U \in \tau$ such that $U \cap F=\varnothing$. But now $U=\bigcup_{i \in \theta}\left\{U \cap W_{i}\right\}$ where $U \cap W_{i} \neq \varnothing$ for each $i \in \theta$ and the sets $U \cap W_{i}$ are disjoint. Let $\tau_{U}=\tau \mid U$ and let $\tau_{i}=\tau_{U}\left|\left(U \cap W_{i}\right)=\tau\right|\left(U \cap W_{i}\right)$.

If it can be shown that each $\tau_{i}$ has a principal complement with an isolated point, then by Theorem $7.4, \tau_{U}$ has a principal complement with an isolated point.

For $i \in \theta, \tau_{i}$ has no isolated points, since if $\{x\} \in \tau_{i}$, then $\{x\}=\left(U \cap W_{i}\right) \cap V$ where $V \in \tau$. But $U \cap V \cap(E-\{x\}) \in \tau$ and $(U \cap V \cap(E-\{x\})) \cap W_{i}=\varnothing$ which contradicts the fact that $W_{i}$ is dense in $\tau$.

If for some $V_{i} \in \tau_{i}, 0<\left|V_{i}\right|<\aleph$, since $\tau_{i} \mid V_{i}$ has no isolated points $\left(i_{i} \in \tau_{i}\right.$ and $\tau_{i}$ has no isolated points), $\tau_{i} \mid V_{i}$ has a principal complement with an isolk. ${ }^{+}$ed point and hence by Theorem 7.5, so does $\tau_{i}$.

If for all $V_{i} \in \tau_{i}, V_{i} \neq \varnothing$ implies $\left|V_{i}\right|=\aleph$, then $\left|U \cap W_{i}\right|=\aleph$. But $U \cap W_{\text {, }}$ is well-ordered (order induced by $E$ ) as $\left\{x_{\beta}\right\}_{\beta<\mu}$ where $\mu$ is the smallest ordinal of cardinality $\aleph$, and since $W_{i}$ has property $P$ with respect to $\tau, U \cap W_{i}$ has property $P$ with respect to $\tau$ and hence with respect to $\tau_{i}$. Therefore by Theorem $7.6, \tau_{i}$ has a principal complement with an isolated point.

Thus $\tau$, has a principal complement with an isolated point for each $i \in \theta$, and by Theorem 7.4, $\tau_{U}$ has a principal complement with an isolated point. Now by Theorem $7.5, \tau$ has a principal complement with an isolated point.

THEOREM 7.8. The lattice of topologies on any set is complemented. Moreover, each topology has a principal complement.

Proof. Every $T_{1}$-topology with no isolated points has a principal complement by Theorem 7.7 , hence by Theorem 7.3 , every $T_{1}$-topology has principal complement. Thus every topology has a principal complement by Theorem 6.3.

\section{REFERENCES}

1. R. W. Bagley, On the characterization of the lattice of topologies, J. London Math. Soc. 30 (1955), 247-249.

2. B. Banaschewski, Über den Ultrafilterraum, Math. Nachr. 13 (1955), 273-281.

3. M.P. Berri, Latticecomplements of certain topologies, Abstract 65T-94 Notices Amer. Math. Soc. 12 (1965), 227.

4. - The complement of a topology for some topological groups, Fund. Math. (to appear).

5. G. Birkhoff, Lattice theory, Amer. Math. Soc. Colloq. Publ. Vol. 25, Amer. Math. Soc., Providence, R. I., 1948. 
6. H. Cartan, Théorie des filtres, C. R. Acad. Sci. Paris 205 (1937), 595-598.

7. O. Fröhlich, Das Halbordnungssystem der topologischen Räume auf einer Menge, Math. Ann. 156 (1964), 79-95.

8. H. Gaifman, The lattice of all topologies on a denumerable set, Abstract 61T-161, Notices Amer. Math. Soc. 8 (1961), 356.

9. - Remarks on complementation in the lattice of all topologies, (unpublished paper).

10. L. Gillman and M. Jerison, Rings of continuous functions, Van Nostrand, New York, 1960.

11. J. Hartmanis, On the lattice of topologies, Canad. J. Math. 10 (1958), 547-553.

12. J. L. Kelley, General topology, Van Nostrand, New York, 1955.

13. Teng-Sun Liu, A note on maximal $T_{1}$-topologies, Portugal. Math. 18 (1959), 235-236.

14. P. Samuel, Ultrafilters and compactifications of uniform spaces, Trans. Amer. Math. Soc. 64 (1948), 100-132.

15. J. Schmidt, Beiträge zür Filtertheorie. I, Math. Nachr. 7 (1952), 359-378.

16. G. Szász, Introduction to lattice theory, Academic Press, New York, 1963.

17. R. Vaidyanathaswamy, Set topology, Chelsea, New York, 1960.

University of New Mexico, Albuquerque, New Mexico

Texas Technological College, LubBock, Texas 